\title{
Personalidad y uso-abuso de cannabis
}

\author{
González, M.P.*; SÁlz, P.A.**; Quirós, M.***; LóPEZ, J.L.**** \\ * Prof. Titular Area de Psiquiatría - Universidad de Oviedo \\ * Prof. Asociado Prof. Titular Area de Psiquiatría - Universidad de Oviedo \\ * * Colaboradora de Honor Area de Psiquiatría - Universidad de Oviedo \\ ***** Prof. Asociado Area de Enfermería - Universidad de Oviedo \\ Enviar correspondencia a: MP González G-Portilla. Universidad de Oviedo \\ Fac. Medicina - Area Psiquiatría. Julián Clavería, 6. 33006 Oviedo
}

\section{Resumen}

Objetivos: determinar los rasgos de personalidad asociados al consumo de cannabis. Método: 3634 reclutas y 2841 estudiantes de secundaria cumplimentaron el EPQ-A y la Escala de Búsqueda de Sensaciones. Cada muestra fue dividida en 3 grupos; G1: no consumidores de sustancias ilegales, G2: consumidores de cannabis, G3: consumidores de cannabis más otras sustancias ilegales. Resultados: Neuroticismo: reclutas: G3 puntuó significativamente más alto que los otros dos grupos; estudiantes mujeres: G1 puntuó significativamente más bajo que los otros dos grupos; estudiantes varones: sin diferencias entre grupos. Intro-extroversión: en las tres muestras G1 puntuó significativamente más bajo que los otros dos grupos. Psicoticismo: estudiantes: diferencias significativas entre los 3 grupos, G3 obtuvo las puntuaciones más altas; reclutas: G3 puntuó significativamente más alto que los otros dos grupos. Búsqueda de sensaciones: las tres muestras obtienen los mismos resultados que los estudiantes en psicoticismo. Conclusiones: existen diferencias de personalidad en función del consumo de cannabis.

Palabras clave: neuroticismo, intro-extroversión, psicoticismo, búsqueda de sensaciones, cannabis.

\section{INTRODUCCIÓN}

E I interés por la personalidad y el usoabuso de sustancias es ya antiguo. Desde los primeros estudios de Eysenck buscando una relación entre el consumo de

\section{Summary}

Objective: to determine the personality traits associated to cannabis consumption. Methods: 3634 military conscripts and 2847 secondary students filledup the EPQ-A and the Sensation Seeking Scale. Each sample was divided into three groups: G1: no consumption of illegal substances; G2: consumption of cannabis; G3: consumption of cannabis plus other illegal substances. Results: Neuroticism: conscripts: G3 scored significantly higher than the other two groups; female students: G1 scored significantly lower than the other two groups; male students: no significant differences across groups. Intro-extroversion: in all samples G1 scored significantly lower than the other two groups. Psychoticism: students: significant differences among the three groups, G3 obtained the highest score; conscripts: G3 scored significanly higher than the other two groups. Sensation seeking: the three samples obtained the same results as students did in pscyhoticism. Conclusions: consumption of cannabis is associated with specific personality traits.

Key words: neuroticism, intro-extroversion, psychoticism, sensation seeking, cannabis, adolescents.

tabaco y la personalidad han sido muchos los investigadores que han dedicado sus esfuerzos a arrojar alguna luz sobre el respecto. Aunque se acepta que no existe una personalidad prototípica del consumidor de sustancias ilegales, existe un elevado interés por 
conocer el papel que juega la personalidad en el desarrollo de la adicción, especialmente de cara a la intervencióni.

Uno de los factores de personalidad que ha sido relacionado con la conducta de usoabuso de cannabis es la "búsqueda de sensaciones"2,3,4. Este rasgo, definido por Zuckerman $^{2,5}$ se caracteriza por la búsqueda de experiencias y sensaciones intensas, novedosas, variadas y complejas, y por la voluntad de experimentar y participar en experiencias que comporten riesgos de diversos tipos (físico, social, legal, ...). En un estudio posterior, Zuckerman ${ }^{6}$ amplia este rasgo de la personalidad pasándolo a denominar "impulsividad no socializada/búsqueda de sensaciones" y considerándolo integrador de los aspectos básicos de la impulsividad de Gray y del psicoticismo de Eysenck. Además, diferencia en él 4 subdimensiones: búsqueda de emociones (aventuras, riesgos); búsqueda de excitación (experiencias); desinhibición; y susceptibilidad al aburrimiento. Diversos autores ${ }^{7,8,4}$, han demostrado la especificidad de estas subdimensiones en cuanto al tipo de droga consumida; el uso de cannabis se asociaba fuertemente a puntuaciones elevadas en la escala de búsqueda de excitación y moderadamente a puntuaciones elevadas en la escala de desinhibición.

En un estudio prospectivo sobre personalidad y uso de cannabis, Shedler y Blocki ${ }^{9}$ encontraron que ya desde la infancia, los chicos que a los 18 años consumían cannabis al menos una vez a la semana y que habían usado al menos otra drogas ("consumidores frecuentes") se diferenciaban del resto. A los 7 años de edad los consumidores frecuentes se caracterizaban por presentar dificultades para planear, poca confianza en sí mismos, eran informales y se mostraban indiferentes a las cuestiones morales. A los 11 años eran distraídos, poco cooperadores, y vulnerables al estrés. A los 18 se caracterizaban por ser inseguros, impulsivos, poco formales, poco considerados, autoindulgentes, e impredecibles en su humor y comportamiento. Ante la frustración reaccionan de forma exagerada, y se sienten inadecuados como personas, y víc- timas y estafados. Serían, en definitiva, adolescentes aislados emocionalemente, infelices, que expresan sus problemas adaptativos mediante conductas abiertamente antisociales. Por el contrario, los "abstinentes" (no habían utilizado drogas ilegales) a los 7 años fueron descritos como inhibidos, convencionales, obedientes, y poco creativos. A los 11 años eran tímidos, limpios y ordenados, y fáciles de contentar pero sin gracia, ni expresividad. A los 18 años eran supercontrolados, moralistas, ansiosos, y poco afectuosos. El "estado ideal" correspondía al grupo de los "experimentadores" (habían utilizado cannabis no más de una vez al mes y habían probado al menos otra droga). Estos ya desde los 7 años eran afectuosos, entusiastas, curiosos, abiertos, activos y alegres. Para estos autores el mecanismo de la adicción al cannabis no sería tanto función de las propiedades psicofarmacológicas intrínsecas del cannabis como de la psicopatología subyacente.

La relación entre la personalidad y el cannabis ha sido estudiada también desde la otra vertiente, no como causa de consumo sino como consecuencia del mismo. Los efectos sobre la salud del uso prolongado del cannabis no son del todo claros. Ello se debe a la escasez de estudios epidemiológicos y a las interpretaciones encontradas de las escasas evidencias existentes, tanto epidemiológicas como de laboratorio ${ }^{10}$. En este sentido, algunos autores han descrito el "síndrome amotivacional" 11 como el resultado del consumo prolongolado de esta sustancia, que se manifestaría especialmente en individuos con trabajo de carácter intelectual ${ }^{12}$ Los sujetos afectos de este síndrome pasarían a ser pasivos, sin objetivos en la vida, apáticos, poco comunicativos, y sin ambiciones. Desde un punto de vista fisiopatológico, los síntomas se explicarían por cambios hormonales, daño cerebral, sedación y depresión. Sin embargo, en la actualidad todavía no está claro que se deba a las propiedades inherentes de la sustancia en vez de a mecanismos de adaptación sociocultural de los consumidores.

Un hallazgo consistente en cuanto a los efectos sobre la salud es la existencia de una 
secuencia en la iniciación en el uso de drogas, en la cual el cannabis típicamente precede a las drogas consideradas como "más duras" como opiáceos y estimulantes ${ }^{13,14}$. Las explicaciones más plausibles ${ }^{10}$ apuntan a que o bien se engancharían al cannabis los adolescentes inconformistas con propensión a utilizar otras drogas ilícitas, o bien que, una vez enganchados al cannabis, la interacción social con pares usuarios de drogas y el mayor acceso al mercado de drogas ilegales aumentarían la probabilidad de usar otras drogas ilícitas.

Con el objeto de aclarar la relación entre la personalidad y el uso-abuso de sustancias ilegales, entre ellas el cannabis, en el Area de Psiquiatría de la Universidad de Oviedo venimos realizando, desde el año 1995, un seguimiento del consumo de sustancias en los jóvenes asturianos.

\section{MATERIAL Y MÉTODOS}

\section{Sujetos}

Los datos del presente estudio se han obtenido de dos poblaciones complementarias, por un lado los jóvenes que se incorporan al Servicio Militar y, por otro, jóvenes estudiantes.
La muestra de los reclutas está constituida por todos los que se incorporaron al Regimiento Príncipe de Asturias (Noreña) en los reemplazos habidos desde el año 1995 al 1999 ( $n=3634)$. La muestra de los estudiantes la forman los alumnos de enseñanza secundaria de los centros públicos de Oviedo que asistieron a clase el día de la encuesta -primavera de 1999- $(n=2841)$.

La edad media de los reclutas era de 20.28 (2.56) años y el $100 \%$ eran varones. En el caso de los estudiantes, la edad media era de 15.87 (1.47), sin que existiesen diferencias estadísticamente significativas en función del sexo (15.85 los hombres frente a 15.89 las mujeres). El 50.9\% eran hombres. En cuanto al nivel de estudios que estaban realizando, el $28.4 \%$ estaba cursando $1^{\circ}$ de bachiller, el $6.1 \% 2^{\circ}$ de la ESO, el $33.8 \% 3^{\circ}$ de la ESO, el $28.9 \% 4^{\circ}$ de la ESO, y el $2.8 \%$ módulos de FP.

\section{Procedimiento e instrumentos de evaluación}

En el caso de los reclutas, los cuestionarios se administraron, sin previo aviso y de acuerdo con los servicios médicos del regimiento, un día de la $1^{\text {a }}$ semana de su incorporación. Tras una breve explicación de los objetivos del estudio para los que solicitábamos

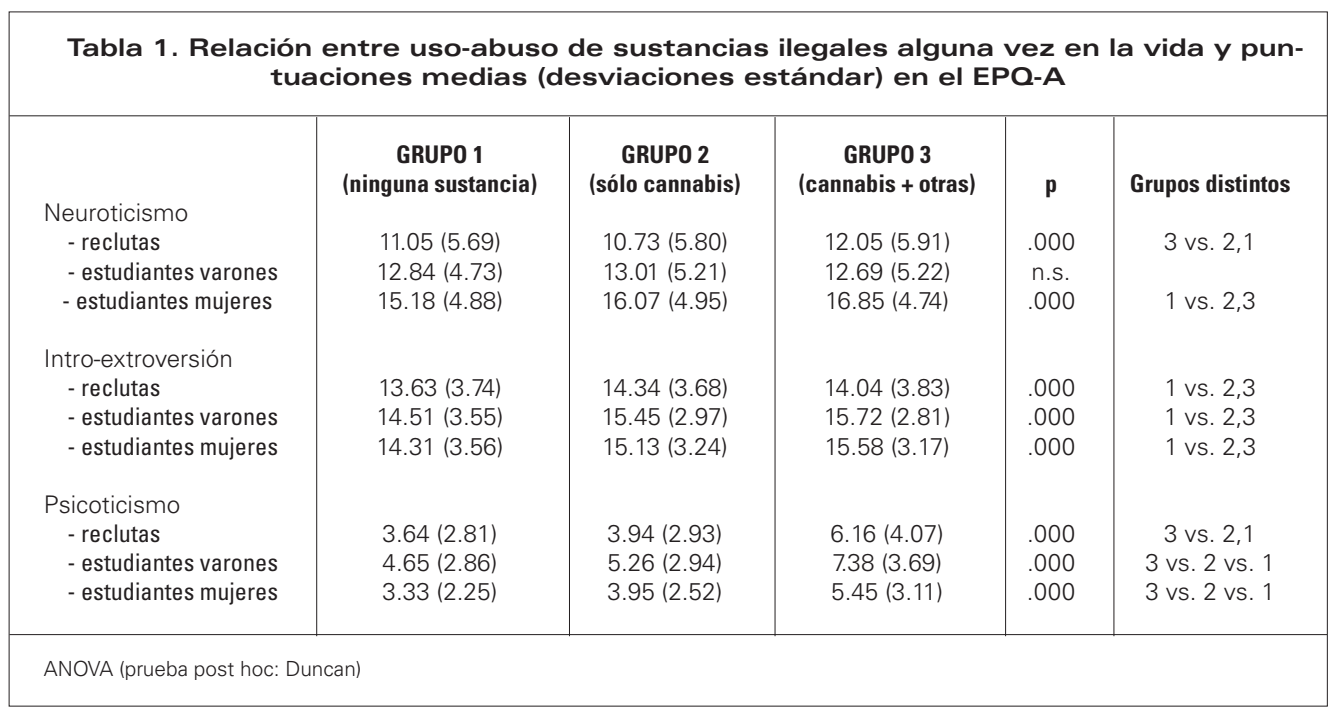




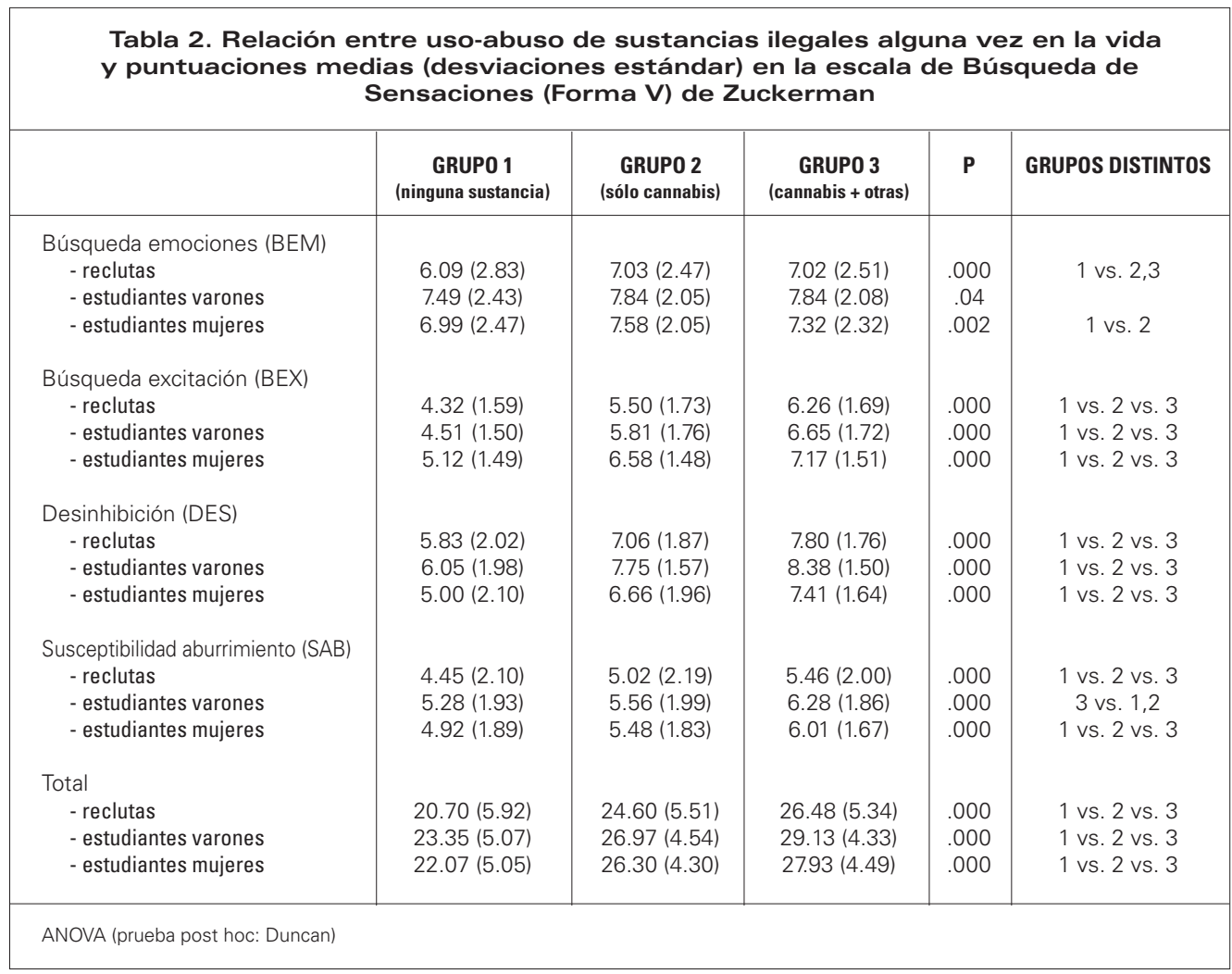

su cooperación y de cómo habían de rellenar las hojas de respuesta, se leían las preguntas en voz alta. Cada recluta contestaba de forma anónima en su hoja de respuestas. Se eligió esta forma de administración (lectura en voz alta) dada la magnitud de los reemplazos, especialmente en los primeros años.

Con los estudiantes se siguió el mismo procedimiento, aunque al ser las clases muy reducidas no se leyeron las preguntas sino que a cada estudiante se le proporcionó el cuadernillo de preguntas y la hoja de respuesta en la que debían contestar.

La encuesta incluía unos mínimos datos sociodemográficos (edad, sexo, estado civil, curso, y situación laboral) y las versiones españolas del Cuestionario de Consumo de Drogas de la $\mathrm{OMS}^{15}$, del Cuestionario de Personalidad de Eysenck para Adultos (EPQ-A) ${ }^{16}$, y de la Escala de Búsqueda de Sensaciones (Forma V) de Zuckerman ${ }^{17}$.
- La Escala de Búsqueda de Sensaciones (Forma V) de Zuckerman

Se trata de un cuestionario originariamente desarrollado en inglés y adaptado a numerosos idiomas, entre ellos el español, diseñado para evaluar la dimensión de la personalidad descrita por Zuckerman como "búsqueda de sensaciones".

Consta de un total de 40 items de respuesta dicotómica sí-no que se agrupan en 4 escalas que corresponden a las 4 subdimensiones de este rasgo de personalidad: búsqueda de emociones (BEM), búsqueda de excitación (BEX), desinhibición (DES), y susceptibilidad al aburrimiento (SAB). Además, de puntuaciones en estas 4 subdimensiones se obtiene una puntuación global de la dimensión general de búsqueda de sensaciones. Obtienen puntuaciones elevadas en esta escala las siguientes poblaciones: deportistas de riesgo, policías, guardaespaldas, delin- 


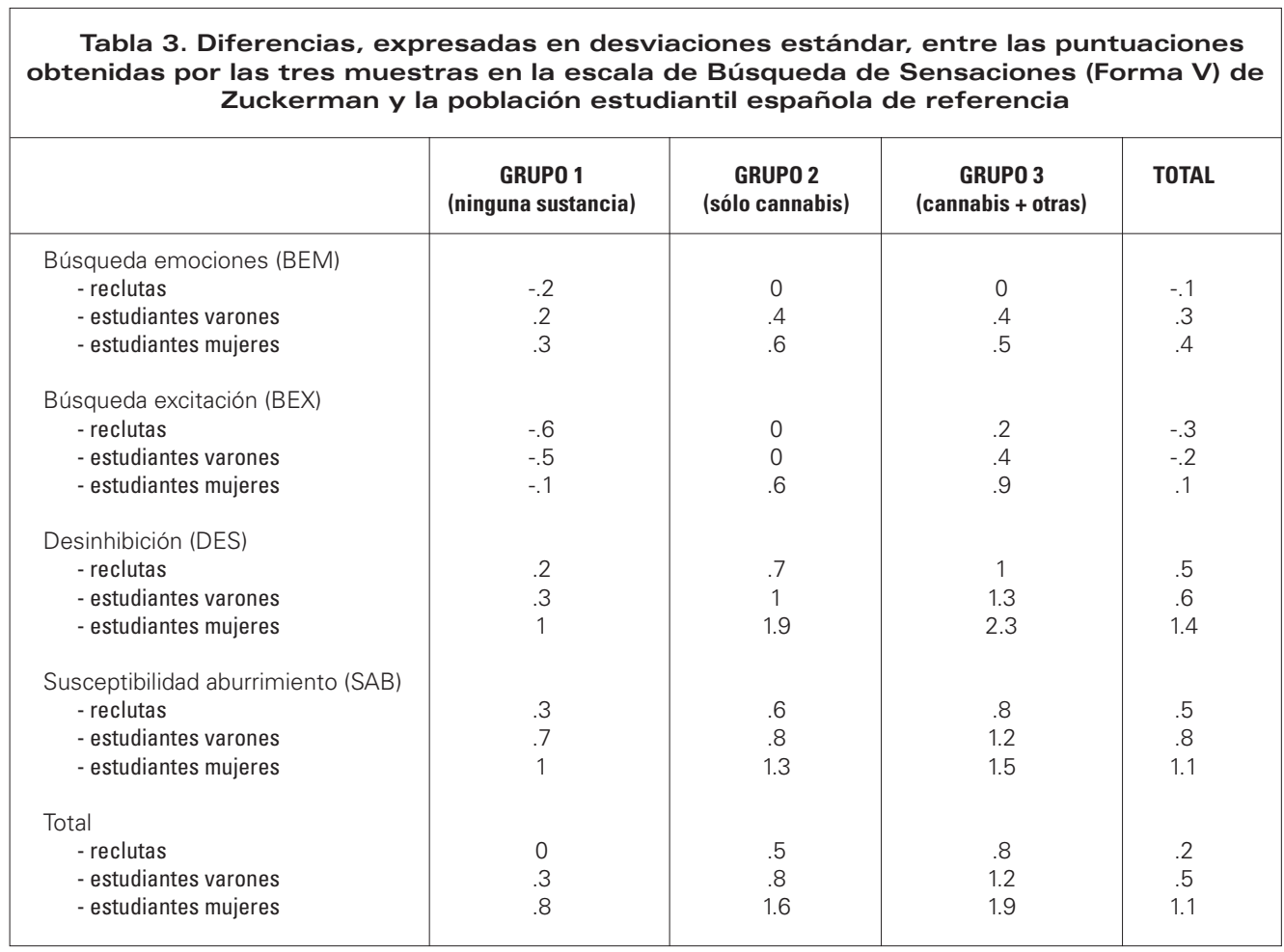

cuentes, consumidores de drogas, y sujetos a los que les gusta poner a prueba sus recursos personales, entre otros.

\section{- El Cuestionario de Personalidad de Eysenck para Adultos}

Al igual que el anterior, se trata de un cuestionario origanariamente desarrollado en inglés que ha sido adaptado y validado en varios idiomas. EI EPQ-A fue desarrollado como fruto del trabajo sobre otros cuestionarios previos. Evalúa las siguientes tres dimensiones básicas de la personalidad: Neuroticismo, Intro-extroversión, y Psicoticismo o Dureza. Además, posee una escala de sinceridad que intenta medir la tendecia al disimulo de algunos sujetos para presentar un "buen aspecto" ${ }^{16}$. Consta de un total de 94 ítems de respuesta dicotómica sí-no; 24 ítems evalúan la dimensión de neuroticismo, 20 la de extro-introversión, 24 la de dureza, y 25 ítems evalúan la sinceridad.

\section{Análisis estadístico}

En la muestra de estudiantes, los datos se analizaron de forma independiente en función del sexo dadas las diferencias en cuanto a personalidad. Por lo tanto, en este estudio se van a presentar los resultados de personalidad y uso-abuso de cannabis de tres muestras: reclutas, estudiantes varones, y estudiantes mujeres. A su vez, cada muestra se dividió en tres grupos en función del consumo: grupo 1 que no había consumido ninguna sustancia ilegal; el grupo 2 que sólo había consumido cannabis como sustancia ilegal; y el grupo 3 que había consumido cannabis más otras sustancias ilegales. Se presentan los datos de las tres muestras y los tres grupos dentro de cada muestra para únicamente una de las tres referencias temporales de consumo que explora el cuestionario de la OMS: alguna vez en la vida.

Además se analizó si existía alguna diferencia en cuanto a personalidad entre los sujetes 


\begin{tabular}{|c|c|c|c|c|c|}
\hline & ABSTINENTES (1) & EXPERIMENTADORES (2) & REINCIDENTES (3) & p & GRUPOS DISTINTOS \\
\hline \multicolumn{6}{|l|}{ Neuroticismo } \\
\hline - reclutas & $11.33(5.71)$ & $11.04(5.92)$ & $12.13(5.73)$ & .001 & 3 vs. 2,1 \\
\hline - estudiantes varones & $12.38(4.82)$ & $13.64(5.15)$ & $12.57(5.13)$ & .01 & 2 vs. 1,3 \\
\hline - estudiantes mujeres & $15.46(4.87)$ & $16.25(4.90)$ & $16.75(4.72)$ & .000 & 1 vs. 2,3 \\
\hline \multicolumn{6}{|l|}{ Intro-extroversión } \\
\hline - reclutas & $13.63(3.73)$ & $14.19(3.74)$ & $14.13(3.74)$ & .000 & 1 vs. 2,3 \\
\hline - estudiantes varones & $14.60(3.53)$ & $14.92(3.35)$ & $15.89(2.61)$ & .000 & 3 vs. 1,2 \\
\hline - estudiantes mujeres & $14.34(3.56)$ & $15.40(3.38)$ & $15.22(3.21)$ & .000 & 1 vs. 2,3 \\
\hline \multicolumn{6}{|l|}{ Psicoticismo } \\
\hline - reclutas & $3.79(2.93)$ & 4.39 (3.36) & $6.22(4.03)$ & .000 & 3 vs. 2 vs. 1 \\
\hline - estudiantes varones & $4.92(3.01)$ & $5.85(3.36)$ & $6.75(3.49)$ & .000 & 3 vs. 2 vs. 1 \\
\hline - estudiantes mujeres & $3.43(2.33)$ & $4.34(2.70)$ & $4.82(2.85)$ & .000 & 3 vs. 2 vs. 1 \\
\hline OVA (prueba post & can) & & & & \\
\hline
\end{tabular}

que tras haber consumido alguna vez en su vida siguieron consumiendo (presentaban consumo también en el último mes) y los que ya no consumían (no había consumos en el último mes). Para ello creamos una nueva variable, suma de consumo alguna vez en la vida y consumo en el último mes, en la que los sujetos se clasificaban en tres grupos: "abstinentes" (nunca consumo, ni alguna vez ni en el último mes); "experimentadores" (consumo alguna vez pero no en el último mes); y "reincidentes" (consumo alguna vez y en el último mes).

Para determinar la significación estadística de las diferencias en las puntuaciones en los cuestionarios de personalidad utilizamos el ANOVA con la prueba Duncan de comparaciones múltiples post hoc, la prueba t de Student, o el cálculo de desviaciones estándar respecto a la norma según fuese necesario. Para el contraste de proporciones se utilizaron las pruebas Chi cuadrado o Z .

\section{RESULTADOS}

\section{Características de consumo de cannabis}

- Consumo de cannabis alguna vez

Contestaron afirmativamente a la pregunta ¿Has consumido alguna vez cannabis (mari- huana, hierba, haschisch o kif)? el $40.4 \%$ de los reclutas frente al $35.3 \%$ de los estudiantes ( $p .000)$ y al $36.6 \%$ de las estudiantes ( $p$. 01). Entre los estudiantes no había diferencias en el consumo en función del sexo.

Al dividir cada una de las muestras en los tres grupos de consumo anteriormente descritos obtuvimos los siguientes resultados: grupo 1 (ninguna sustancia ilegal) el 58.6\% de los reclutas, el $63.3 \%$ de los estudiantes, y el $62.6 \%$ de las estudiantes; grupo 2 (sólo cannabis): el $18.8 \%$ de los reclutas, el $17.1 \%$ de los estudiantes, y el $21.7 \%$ de las estudiantes; y grupo 3 (cannabis más otras sustancias ilegales) el $22.6 \%$, el $19.6 \%$, y el $15.7 \%$ de los reclutas, estudiantes varones, y estudiantes mujeres respectivamente.

\section{- Persistencia del consumo (consumo de cannabis alguna vez y en el último mes)}

Eran "abstinentes", es decir, no habían consumido cannabis ni alguna vez ni en el último mes, el $60.7 \%$ de los reclutas, el $64.9 \%$ de los estudiantes, y el $64.3 \%$ de las estudiantes. Contestaron como "experimentadores", consumo alguno vez pero no en el último mes, el $21.6 \%$ de los reclutas, el $11.7 \%$ de los estudiantes, y el $16 \%$ de las estudiantes. Finalmente, fueron considerados "reinciden- 


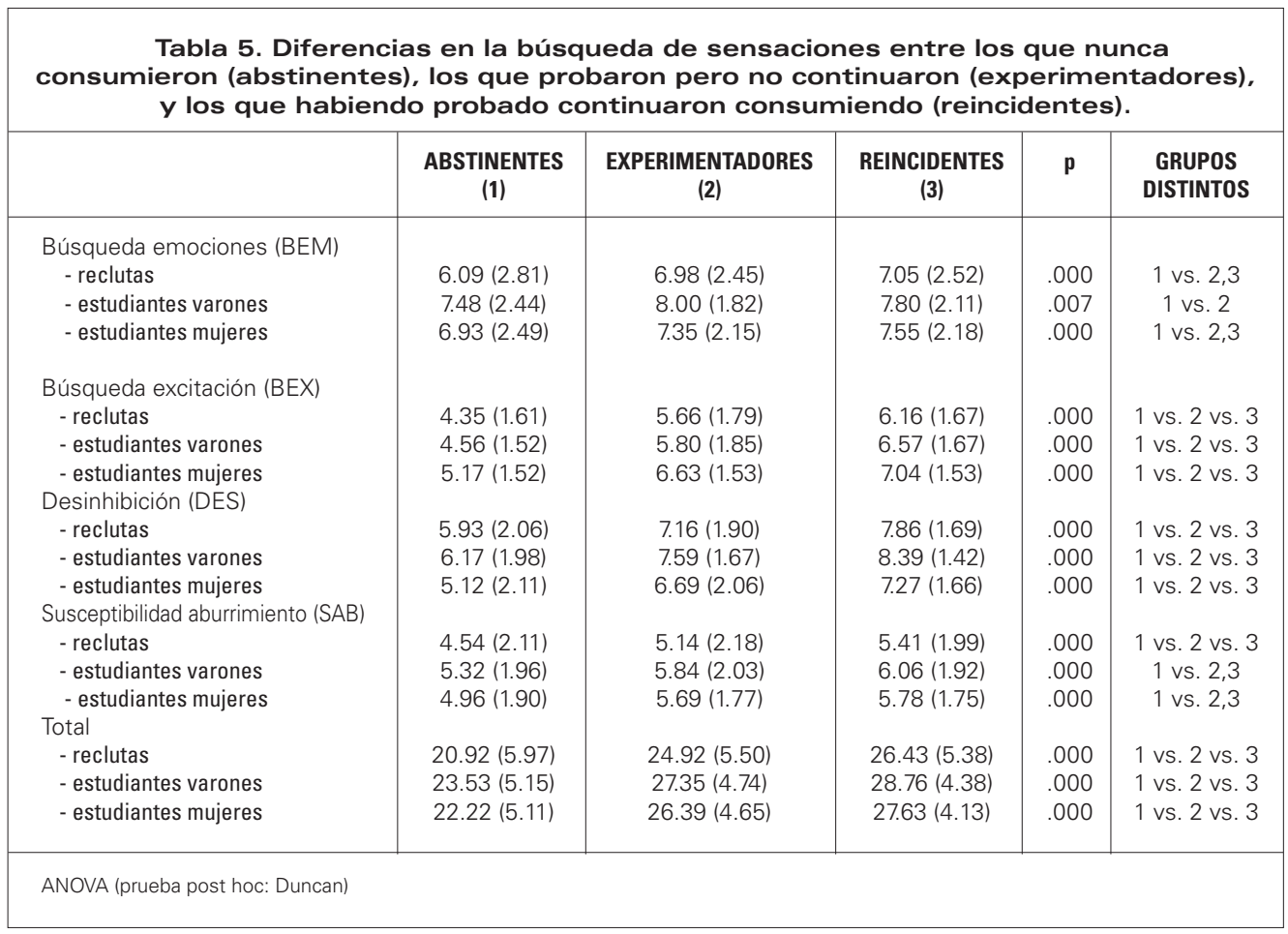

tes" (consumos alguna vez y en el último mes) el $17.7 \%$ de los reclutas, el $23.3 \%$ de los estudiantes, y el $19.7 \%$ de las estudiantes.

\section{Características de personalidad}

- Cuestionario de Personalidad de Eysenck (EPQ-A)

La puntuación media obtenida por los reclutas en las 3 dimensiones del EPQ-A fueron 11.22 (5.77) en neuroticismo, 13.85 (3.76) en extroversión, y 4.26 (3.32) en psicoticismo. En el caso de los estudiantes varones las puntuaciones fueron: 12.49 (4.91) en neuroticismo, 14.90 (3.36) en extroversión, y 5.29 (3.23) en psicoticismo. Los estudiantes puntuaron significativamente ( $p$.0000) más alto que los reclutas en las tres dimensiones. Estas diferencias estadísticas, sin embargo, sólo tienen trascendencia clínica en el caso del psicoticismo. Los estudiantes se sitúan en el rango de elevado psicoticismo (percentil 85) mientras que los reclutas lo hacen en el de normalidad-alto (percentil 80). Las muje- res estudiantes obtuvieron las siguientes puntuaciones: 15.63 (4.91) en neuroticismo, 14.69 (3.47) en extroversión, y 3.79 (2.58) en psicoticismo. Clínicamente, en las tres dimensiones se sitúan en el rango de la normalidad.

\section{- Escala de Búsqueda de Sensaciones (Forma V) de Zuckerman}

En la escala de búsqueda de sensaciones de Zuckerman los reclutas obtuvieron las siguientes puntuaciones: 6.47 (2.73) en búsqueda de emociones, 4.98 (1.84) en búsqueda de excitación, 6.51 (2.11) en desinhibición, 4.79 (2.13) en susceptibilidad al aburrimiento, y 22.74 (6.24) en total. Las puntuaciones de los estudiantes varones fueron: BEM 7.62 (2.31), BEX 5.15 (1.82), DES 6.80 (2.08), SAB 5.52 (1.96) y total 25.10 (5.40). Los estudiantes varones puntuaron significativamente más alto que los reclutas en las 4 escalas y, consecuentemente, en la puntuación total. Al comparar las puntuaciones de estas dos 


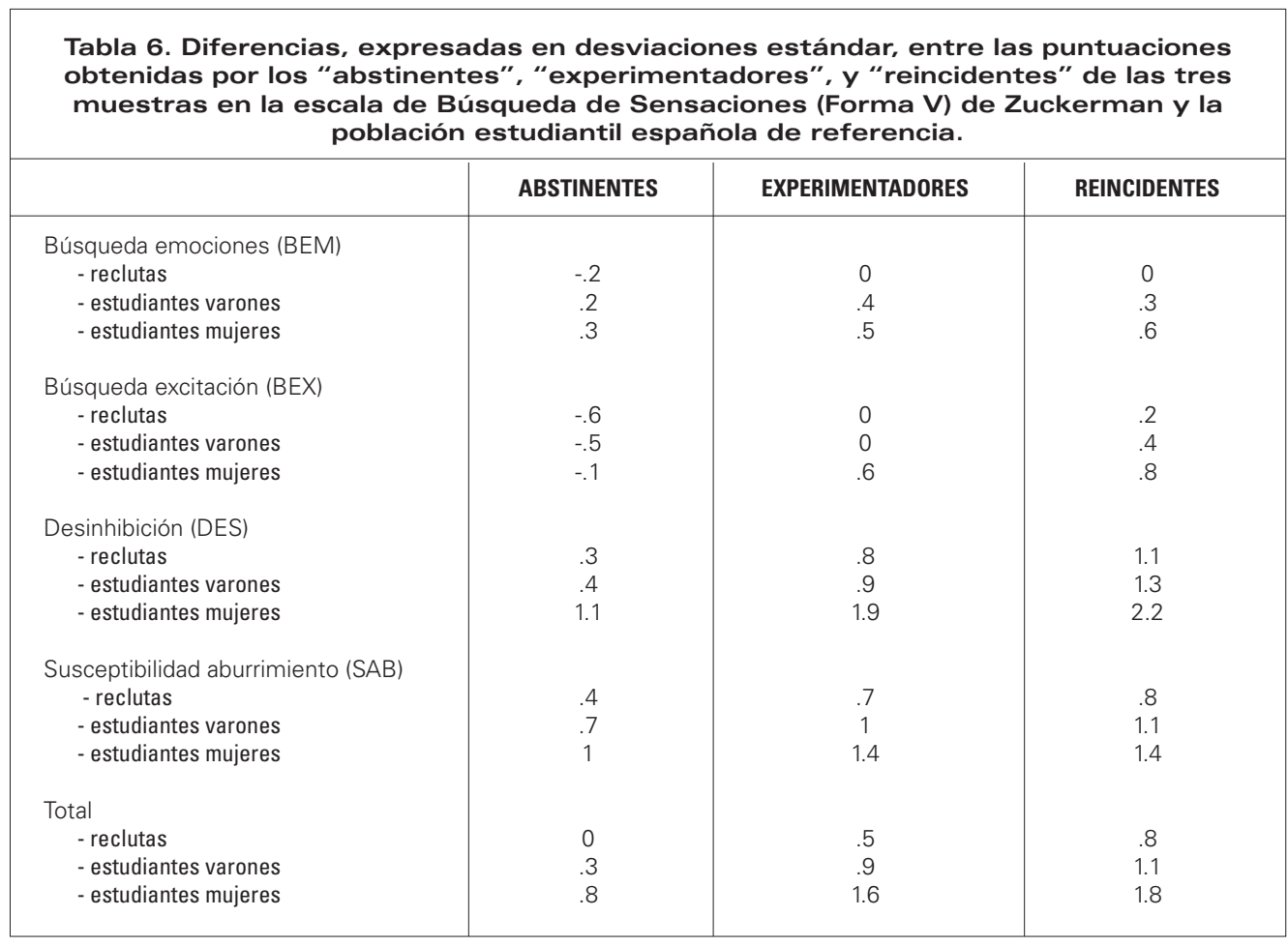

muestras con las de los valores de la población de estudiantes varones españoles de referencia ${ }^{17}$ encontramos diferencias estadísticamente significativas en todas las escalas, excepto en la escala BEM en el caso de los reclutas. Cuando calculamos las diferencias en términos de desviación estándar, la desviación máxima fue de +0.87 en la escala $\mathrm{SAB}$ en la muestra de estudiantes.

Las puntuaciones que obtuvieron las mujeres estudiantes fueron: 7.17 (2.37) en la escala BEM, 5.75 (1.71) en la BEX, 5.74 (2.24) en la DES, 5.21 (1.89) en la SAB, y 23.91 (5.39) como puntuación total. Comparadas con las puntuaciones de las estudiantes españolas de referencia ${ }^{17}$, puntuaron significativamente más alto en todas las escalas y en la puntuación total. Cuando traducimos estas diferencias estadísticas en desviaciones con respecto a la norma encontramos que en tres escalas las desviaciones fueron superiores a 1. En la escala DES nuestras estudiantes se encuentran 1.44 desviaciones estándar por encima de la norma; en la escala SAB se encuantran 1.16 d.e. por encima; y en la puntuación total en la escala 1.17 d.e. por encima.

Consumo de cannabis alguna vez en la vida y personalidad

La tabla 1 muestra los resultados obtenidos en el análisis de la la asociación entre uso-abuso de sustancias alguna vez en la vida y el EPQ-A. Los resultados obtenidos en la dimensión de neuroticismo varían de una muestra a otra: en la muestra de los reclutas el grupo 3 (e.d., consumidores alguna vez de cannabis y otras sustancias ilegales) obtuvo puntuaciones significativamente más elevadas que los otros dos grupos; en la muestra de estudiantes varones no se encontraron diferencias estadísticamente significativas entre los tres grupos; $y$, finalmente, en la muestra de estudiantes mujeres el grupo 1 (no consumo de sustancias ilegales) obtuvo puntuaciones significativamente inferiores a las de los otros dos grupos. 
En la dimensión de intro-extroversión los resultados fueron unánimes: en las tres muestras los sujetos que nunca consumieron sustancias ilegales obtuvieron puntuaciones significativamente inferiores a las de los otros dos grupos.

Finalmente, en la dimensión de psicoticismo en las dos muestras de estudiantes (hombres y mujeres) los 3 grupos son significativamente distintos entre sí, de tal manera que se puede hablar de un gradiente de psicoticismo en función del consumo: a más sustancias mayor puntuación en psicoticismo. En la muestra de los reclutas los grupos 1 y 2 eran iguales, mientras que el grupo 3 puntuaba significativamente más alto que los otros dos. En cualquier caso, el grupo 3 obtuvo puntuaciones significativamente más altas que los otros dos grupos en las tres muestras.

Desde un punto de vista clínico, los resultados en el EPO-A sugieren que el hecho de no consumir sustancias ilegales, consumir tan sólo cannabis, o consumir cannabis más otras sustancias ilegales se asocia a diferencias de personalidad, de tal manera que a medida que el consumo es más importante aumentan los niveles de neuroticismo, extroversión, y psicoticismo. Sin embargo, es necesario señalar, que tan sólo la dimensión de psicoticismo adquiere trascendencia clínica, ya que es en la única en la que tanto los consumidores de sólo cannabis como los que además consumen otras sustancias se sitúan en rangos iguales o superiores al percentil 85.

La relación entre el uso-abuso de sustancias ilegales alguna vez en la vida y la escala de búsqueda de sensaciones de Zuckerman se reflejan en la tabla 2 . En conjunto, se puede decir que en las tres muestras las puntuaciones aumentan significativamente en función del consumo, de tal forma que son más bajas en el grupo que no consume sustancias ilegales y más altas en el grupo 3 que consume cannabis más otras sustancias ilegales. Estas diferencias no se dan en la escala de búsqueda de emociones. Esta escala BEM se comporta de distinta forma en las tres muestras: en los reclutas el grupo 1 puntúa significativamente menos que los otros dos grupos; en los estudiantes varones no hay diferencias estadísticamente significativas entre los tres grupos; y en las estudiantes el grupo 1 puntúa significativamente menos que el grupo 2.

Desde el punto de vista clínico, este instrumento se comporta de igual modo que el anterior, mayor desviación respecto a la norma en función de la importancia del consumo (tabla 3). No obstante, la trascendencia clínica es escasa: únicamente en la escala de desinhibición el grupo 3 de la muestra de estudiantes mujeres se diferencia de su población de referencia (estudiantes mujeres españolas) 2 ó más desviaciones estándar (2.3 d.e.).

Persistencia en el consumo: Diferencias de personalidad entre los abstinentes, los experimentadores, y los reincidentes

En la tabla 4 se presentan las puntuaciones obtenidas en el EPQ-A por las tres muestras estudiadas en función de la frecuencia del consumo de cannabis. Los resultados obtenidos son casi una réplica de los mostrados en la tabla 1 referentes a la asociación entre personalidad y sustancias ilegales consumidas. En la dimensión de neuroticismo aunque no hay unanimidad en cuanto a qué grupos difieren de qué otros entre las tres muestras, lo que se observa es una tendencia a que los sujetos reincidentes obtengan puntuaciones superiores. En la dimensión de intro-extroversión en las muestras de reclutas y de estudiantes mujeres el grupo 1 (abstinentes) es significativamente más introvertido que los otros dos grupos, mientras que en la de estudiantes varones el grupo de reincidentes es significativamente más extrovertido que los otro dos grupos. Finalmente, existe unanimidad en los resultados en la dimensión de psicoticismo; en las tres muestras se observa un gradiente de tal modo que las puntuaciones aumentan significtivamente de un grupo a otro, siendo los abstinentes los que significativamente puntúan más bajo y los reincidentes los que significativamente puntúan más alto.

Nuevamente, estas diferencias estadísticas tan sólo tienen significado clínico en la dimensión de psicoticismo; en las tres muestras los 
abstinentes se encuentran en percentiles de normalidad, los experimentadores en percentiles límite, y los reincidentes en percentiles clínicamente significativos (superiores a P85).

Los resultados de la asociación entre la búsqueda de sensaciones y el seguir consumiendo cannabis se muestran en la tabla 5 . Las tres muestras, en las escalas búsqueda de excitación, desinhibición, y puntuación total, presentan de nuevo un gradiente en las puntuaciones de tal modo que a mayor persistencia del consumo mayores puntuaciones. En las otras dos escalas, con la excepción de los reclutas en la escala de susceptibilidad al aburrimiento, en las tres muestras el grupo de abstinente obtuvo puntuaciones significativamente menores que los otros dos grupos, entre los cuales no existían diferencias significativas.

Desde el punto de vista clínico (tabla 6), otra vez son las escalas de desinhibición y susceptibilidad al aburrimiento las que mayores desviaciones presentan respecto a la norma, llegando a estar las estudiantes reincidentes 2.24 desviaciones estándar por encima de la norma en la escala DES.

Sin embargo, en estos datos de personalidad y persistencia en el consumo del cannabis es necesario tener en cuenta la presencia de una variable confusora: el consumo de otras sustancias. En los análisis anteriores sobre consumo de sustancias alguna vez en la vida y personalidad, habíamos visto cómo los rasgos de personalidad se iban acentuando en función de lo que consumían; nada, sólo cannabis, y cannabis más otras sustancias ilegales. Al tener en cuenta esta variable, hemos observado que, en las tres muestras, el grupo de reincidentes había consumido alguna vez cannabis más otras sustancias ilegales significativamente en mayor proporción que el grupo de los experimentadores (reclutas: $75.4 \%$ vs 38.3\%; estudiantes varones: $59.1 \%$ vs $42.9 \%$; estudiantes mujeres: $48.5 \%$ vs $34.2 \%$, p .000). Luego, puede que las diferencias de personalidad que en principio hemos encontrado asociadas a la persistencia del consumo se deban, al menos en parte, a que los sujetos del grupo reincidente son los que consumen más sustancias.

\section{DISCUSIÓN}

Los resultados obtenidos en el presente estudio indican la existencia de una asociación entre rasgos de personalidad y consumo de cannabis en los adolescentes. Así, encontramos que, a medida que se agrava cualitativa y cuantitativamente el consumo de sustancias ilegales progresivamente se acentúan los rasgos de personalidad, llegando incluso a alcanzar niveles clínicamente significativos, como en el caso de la dimensión de psicoticismo.

En el EPQ-A, los resultados varían en cada dimensión. En el neuroticismo es donde los resultados son menos claros. Así, mientras que en los reclutas los consumidores de cannabis más otras sustancias son más neuróticos que los otros dos grupos que no se diferencian entre sí, en las estudiantes los que no consumen sustancias ilegales son menos neuróticos que los dos grupos que consumen sustancias ilegales, y, finalmente, en los estudiantes no existen diferencias entre los tres grupos. En la dimensión de intro-extroversión existe unanimidad absoluta, los no consumidores son más introvertidos que los otros dos grupos que no se diferencian entre sí. Por último, en el psicoticismo, en los estudiantes (hombres y mujeres) los tres grupos difieren entre sí: los no consumidores presentan puntuaciones más bajas que los consumidores de cannabis, y éstos a su vez obtienen puntuaciones más bajas que los consumidores de cannabis más otras sustancias. En los reclutas los consumidores de cannabis más otras sustancias son los que se diferencian de los otros dos grupos que son iguales entre sí.

En la Escala de Búsqueda de Sensaciones podríamos decir que a mayor gravedad de consumo mayor acentuación de este rasgo, resultado que cabría esperar ya que este rasgo de la personalidad está incorporado en la dimensión de psicoticismo de Eysenck ${ }^{18}$. Sin embargo, con respecto a estos resulta- 
dos, consideramos necesario hacer la siguiente consideración. Los datos de la población de referencia utilizados, claves para determinar la acentuación del rasgo ya que ésta es expresión de la desviación respecto a la norma, fueron publicados en el año 1985. Creemos que los cambios sociales ocurridos en el transcurso de al menos 14 años (desde que fueron publicados hasta que administramos los cuestionarios a nuestros alumnos) podrían influir de algún modo en magnitud de las desviaciones con respecto a la norma. Incluso los estudiantes que nunca en su vida han consumido ninguna sustancia ilegal presentan desviaciones con respecto a la norma considerables, especialmente en el caso de las mujeres. Por ejemplo, las estudiantes que nunca han consumido sustancias ilegales presentan una desviación de +.8 en la puntuación total en esta escala. Claro está, por otra parte, que las estudiantes que han consumido cannabis más otras sustancias duplican esta desviación con respecto a la norma, ya que obtienen puntuacionese que representan +1.9 desviaciones estándar. En conclusión, pensamos que sí existe una acentuación del rasgo en función del consumo pero no de magnitud tan grande como los datos sugieren.

La relación entre los rasgos de personalidad del modelo PEN de Eysenk y el uso de sustancias legales (tabaco) ha sido evidenciada en distintos estudios apuntando a mayores niveles de neuroticismo y extroversión en los fumadores ${ }^{19,20,21,22}$. Los sujetos con elevados niveles de neuroticismo utilizarían el tabaco como un mecanismo para reducir su ansiedad, especialmente en los sujetos introvertidos. Si además, este rasgo está presente en el contexto ambiental del consumo de sustancias ilegales la evitación de estas conductas antinormativas sería pasiva, siendo por tanto un factor modulador del consumo de drogas ${ }^{22}$. Pensamos que estos mismos mecanismos podrían estar implicados la relación entre los elevados niveles de neuroticismo y el consumo de cannabis encontrados en este estudio y en un estudio previo ${ }^{23}$. Del mismo modo, la extroversión sería otra varia- ble que mediaría el consumo a través de la fuerte necesidad que tienen estos sujetos de excitación. Sin embargo, el rasgo que parece ser el predictor más potente de consumo de sustancias ilegales es la búsqueda de sensaciones $^{24,25}$, siendo mejor predictor que la ansiedad, el humor depresivo, que el MMPI o que el MCMI a la hora de identificar a los no consumidores, los consumidores experimentales, y los consumidores de todo tipo de drogas.

Los estudios específicos sobre rasgos de personalidad y consumo de cannabis son escasos, y los existentes se centran más en el rasgo de búsqueda de sensaciones e impulsividad, que como ya hemos comentado anteriormente estarían representados por la dimensión de psicoticismo de Eysenck. Los estudios sobre este rasgo ponen de manifiesto una elevación de las subdimensiones de búsqueda de excitación y desinhibición ${ }^{8,74,}$ así como un aumento de la impulsividad $^{9}$. En nuestro caso, las escalas que más diferencia presentan respecto a la norma son las de desinhibición y la de susceptibilidad al aburrimiento. Sin embargo, volvemos a enfatizar en lo dicho al principio de este apartado. Si nos fijamos en la tabla 3, aunque los datos indiquen que la escala SAB está más alejada de la norma que la escala BEX, si hallamos las diferencias que existen en cada una de las dos escalas entre los tres grupos vemos que existe una mayor acentuación de la subdimensión BEX que de la subdimensión SAB en función del consumo. En la escala BEX el pertenecer al grupo 2 (sólo consumidores de cannabis) implica un aumento de 0.6 desviaciones estándar en la muestra de los reclutas, de 0.5 d.e. en la muestra de los estudiantes, y de 0.7 en la de las estudiantes. Por el contrario, en la escala SAP los incrementos son menores; 0.3 en los reclutas, 0.1 en los estudiantes, y 0.3 en las estudiantes. En el paso entre el grupo 2 y el grupo 3 (consumir cannabis más otras sustancias ilegales) las dos escalas únicamente difieren en la muestra de las estudiantes, incrementándose la diferencia con respecto a la norma en 0.3 d.e. en la escala BEX y en 0.2 en la escala SAB. 


\section{BIBLIOGRAFÍA}

(1) Calafat Far A, Amengual Munar M, Farres Snelders C, Palmer Pol A: Estilo de vida y hábitos de consumo de drogas entre estudiantes de enseñanza media. Boletín de Estupefacientes 1985; XXXVII(2-3): 121-131.

(2) Zuckerman M: Sensation seeking: beyond the optimal level of arousal. Hillsdale: NJ Erlbaum. 1979.

(3) Brill NW, Crumpton E, Grayson HM: Personality factors in marijuana use: a preliminary report. Arch Gen Psychiatry 191; 24: 163-165.

(4) Pedersen W, Clausen SE, Lavik NJ: Patterns of drug use and sensation-seeking among adolescents in Norway. Acta Psychiatr Scand 1989; 79: 386-390.

(5) Zuckerman M: Biologiacal expressions and biosocial bases of sensation seeking. Nueva York: Cambridge Univ. Press. 1994.

(6) Zuckerman M, Kuhlman DM, Joireman J, Teta P: A comparison of three structural models for personality: the big three, the big five, and the alternative five. J Pers Soc Psychol 1993; 65(4): 757-768.

(7) Huba GJ, Newcomb MD, Bentler PM: Comparison of canonical correlation and interbattery factor analysis on sensation seeking and drug use domain. Appl Psychol Measure 1981; 5: 291-306.

(8) Mabry EA, Khavari KA: Attitude and personality correlates of hallucinogenic drug use. Int $\mathbf{J}$ Addict 1986; 21: 691-699.

(9) Shedler J, Block J: Adolescent drug use and psychological health: a longitudinal inquiry. Am Psychologist 1990; 45: 612-630.

(10) Hall W, Solowij N: Adverse effects of cannabis. The Lancet 1998; 352: 1611-16.

(11) McGlothlin WH, West LJ: The marijuana problem: an overview. Am J Psychiatry 1968; 125: 370-378.

(12) Grinspoon L, Bakalar JB: Marihuana. En: Lowinson JH, Ruiz P, Millman RB, Langrod JG, eds. Substance Abuse. A Comprehensive Textbook (3rd edi.) Baltimore: Williams \& Wilkins. 1997.

(13) Kandel DB, Davies M: Progression to regular marijuana involvement: phenomenology and risk factors for near daily use. En: Gantz M, Pickens R, eds. Vulnerability to Drug Abuse. Washington, DC: American Psychological Association. 1992.

(14) Yamaguchi K, Kandel DB: Patterns of drug use from adolescence to adulthood: II-sequences of progression. Am J Public Health 1984; 74: 668-72.

(15) Smart RG, Hughes PH, Johnston LD, Anumonye A, Khant U, Medina ME, Navaratnam V, Poshyachinda V, Varma VK, Wadud K: Méthodologie pour des enquêtes sur l'usage des drogues chez les étudiants. Publication offset, $n^{\circ}$ 50. Genève: OMS. 1980.

(16) Eysenck HJ, Eysenck SBG: EPQ. Cuestionario de personalidad para niños (EPQ-J) y adultos (EPQ-A). Manual. (7 ${ }^{a}$ edi.). Madrid: TEA. 1995.

(17) Pérez J, Torrubia R: Fiabilidad y validez de la versión española de la Escala de Búsqueda de Sensaciones (Forma V). Revista Latinoamericana de Psicología 1986; 18(1): 7-22.

(18) Andrés Pueyo, A: Manual de Psicología Diferencial. Madrid: McGraw-Hill. 1997.

(19) Eysenck HJ, Tarrant M, Woolf M, England L: Smoking and personality. Brit Med J 1960; 1 : 1456-1460.

(20) Frith CD: Smoking behaviour and its relations to the smokers' immediate experience. Brit J Soc Clin Psychol 1971; 10: 73-78.

(21) Parkes KR: Smoking and the Eysenck personality dimensions: an interactive model. Psychol Medicine 1984; 14: 825-834.

(22) Pérez J, García-Sevilla L: Neuroticismo, extraversión y consumo de tabaco. Psiquis 1986; VII: 122-128.

(23) Sáiz PA, González MP, Jiménez L, Delgado Y, Liboreiro MJ, Granda B, Bobes J: Consumo de alcohol, tabaco, y otras drogas y rasgos de personalidad en jóvenes de enseñanza secundaria. Adicciones 1999; 11(3): 209/220.

(24) Jaffe LT, Archer RP: The prediction of drug use among college students from MMPI, MCMI, and sensation seeking scales. J Pers Assess 1987; 51: 243-253.

(25) Teichman M, Barnea Z, Rahav G: Sensation seeking, state and trait anxiety, and depressed mood in adolescent substance users. Int $\mathbf{J}$ Addict 1989, 24(2): 87-99. 